\title{
COVID-19 therapies and vaccine landscape
}

\author{
Within a few weeks of the novel coronavirus genome sequence being published, numerous therapies and vaccines \\ have entered clinical trials with a few showing great promise in alleviating symptoms and accelerating recovery.
}

n late 2019, patients presenting with viral pneumonia were reported in Wuhan, China, and a novel coronavirus was quickly identified as the causative pathogen that leads to severe acute respiratory syndrome (SARS-CoV-2) or COVID19 . The widely reported unpredictability of the disease means that some infected individuals are asymptomatic, some have mild flu-like symptoms, whereas other unfortunate individuals suffer from major complications. The lower respiratory tract is the main target for severe symptoms, where high virus shedding has been observed. The body defends itself by an elevated immune response that fights to destroy virus-infected cells. This unfortunately results in blockage of the alveoli in the lungs due to inflamed tissue, leading to shallow respiration, with some patients presenting with a condition called acute respiratory distress syndrome that can cause death ${ }^{1}$. As of July 2020, there have been over 10 million confirmed cases of coronavirus infections and over 500,000 deaths globally. The emergence of the virus is still contested, although it is now known that it is phylogenetically related to the SARS-like coronavirus isolated from horseshoe bats (Rhinolophus sinicus) ${ }^{2}$. Unlike SARS and MERS (Middle Eastern respiratory syndrome), other zoonotic coronavirus-based diseases, COVID-19 has been declared a pandemic by the World Health Organization (WHO).

Although currently much hope rests in the development of a vaccine, there has been noteworthy progress in repurposing existing broad-spectrum antiviral agents ${ }^{3}$. Writing in a Comment in this issue of Nature Materials, Nam Joon Cho and Jeffrey Glenn highlight that numerous viruses share similar physical structures and lifecycle patterns. Accordingly, a number of existing antiviral drugs are now being used for the treatment of the coronavirus. Remdesivir (Gilead Sciences, Inc.) is one such drug that was previously developed for treating Ebola and Marburg viruses. It is currently being explored for COVID-19 and has reached phase III clinical trials. Preliminary results have shown that patients with moderate COVID-19 pneumonia receiving the drug are $65 \%$ more likely to have clinical improvement when compared to those on standard care plans ${ }^{4}$. Another drug,

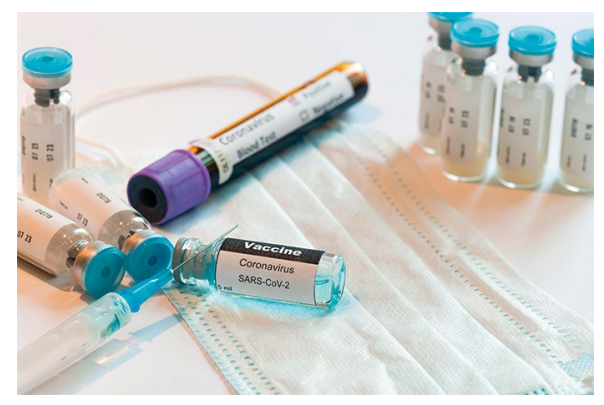

Credit: Andrey Deryabin/Alamy Stock Photo

Favipiravir (Toyama Chemical), previously developed for the treatment of influenza, has also been trialled for COVID-19, and shows the potential to reduce viral load and offer clinical improvement in patients with mild to moderate symptoms of COVID-19. Other drugs such as Lopinavor, Ritonavir and even hydroxycholoroquine have also been tested but have been less successful.

A rather surprising but positive outcome has been seen with corticosteroids, which were previously thought to limit the body's ability to fight the virus but in fact have been crucial in alleviating symptoms associated with pulmonary failure. In a recent randomized, controlled clinical trial (RECOVERY trial) in the UK involving over 11,500 patients, the use of low-dose dexamethasone, a corticosteroid, was shown to reduce deaths by approximately $30 \%$ in COVID-19 patients on ventilators and by $20 \%$ in patients on oxygen alone $e^{5}$. It is thought that this low-cost drug suppresses the immune system in severely ill patients whose lungs are compromised by inflammation. Ultimately, a vaccine would offer more effective protection from the virus.

Vaccines typically take $5-10$ years to develop and the process involves highly controlled preclinical and clinical trials before approval for general use. Incredibly, within a few weeks of the virus genome sequence being published, vaccines were already developed for patient trials. Indeed, there are over 130 COVID-19 vaccines at various stages of development around the world ${ }^{6}$. In another Comment, Debby van Riel and Emmie de Wit highlight that next-generation vaccine development can be expedited by utilizing sequence information alone rather than relying on in vitro cultures of live viruses. Nucleic acid-based vaccines that use this next-generation approach have been the frontrunners for COVID-19. One such example is an mRNA vaccine (mRNA1273 - encoding for the virus spike protein used by viruses to latch onto human host cells) developed by Moderna Therapeutics and the National Institutes of Health has already shown promising signs and is currently finalizing preparations for phase III clinical trials ${ }^{7}$. Another frontrunner in phase IIB/III clinical trials, ChAdOX1 nCoV19, developed by Oxford University and AstraZeneca, is a vaccine platform based on an adenovirus vector also encoding for the spike protein of SARS-CoV-2 ${ }^{8}$. This platform was previously developed for MERS and influenza and has also shown significant promise thus far for COVID-19. Scale-up manufacture of these candidates will be a major hurdle in the near future.

Equally important, diagnosis of patients is instrumental in the fight against COVID19 and so far, real-time polymerase chain reaction (RT-PCR) has been the most successful at detecting the virus. However, other diagnostic toolkits are also in development. Antigen and antibody tests have been recommended by WHO for the detection of the virus at early and late stages of the disease, respectively, and numerous pharmaceutical companies are developing a range of rapid toolkits.

Overall, it is clear that the landscape of vaccines, therapeutics and diagnostic tools for COVID-19 is evolving rapidly with promising preliminary results already, which in itself is a significant achievement.

Published online: 23 July 2020 https://doi.org/10.1038/s41563-020-0758-9

\footnotetext{
References

1. Wadman, M., Couzin-Frankel, J., Kaiser, J. \& Matacic, C. Science http://doi.org/ggsd2f (2020).

2. Zhou, P. et al. Nature 579, 270-273 (2020).

3. Harrison, C. Nat. Biotechnol. 38, 379-381 (2020).

4. Beigel, J. H. et al. N. Engl. J. Med. http://doi.org/dwkd (2020).

5. University of Oxford https://go.nature.com/2O3HeqY (2020).

6. Mullard, A. Lancet 395, 1751-1752 (2020).

7. Moderna https://go.nature.com/3faEy6Z (2020).

8. University of Oxford https://go.nature.com/3e7XUYV (2020).
} 